\title{
Effects and Mechanisms of Interaction of Electromagnetic Fields on Cells
}

\author{
David Gómez-Rios ${ }^{1 *}$ \\ 1 Grupo de Bioprocesos, Departamento de Ingeniería Química, Universidad de Antioquia UdeA, Calle 70 No. \\ 52-21, Medellín, Colombia; dandres.gomez@udea.edu.co \\ * Correspondence: dandres.gomez@udea.edu.co
}

\begin{abstract}
Modern life implies a constant exposure of living organisms to electromagnetic fields generated by human made technology. The question of whether or not electromagnetic fields in the non-ionizing frequency range can affect cellular functions, increasing the risk of cancer or another pathologies is currently a subject of interest for scientific community of several disciplines of physics, biology, chemistry and medicine. The first part of this short review presents briefly the possible mechanism of interaction of electromagnetic fields in cellular level based in theoretical models and experimental results. The second part refers to experimental observations published by several authors about the potential cytotoxic and genotoxic effects of electromagnetic fields. Results of researches are no yet conclusive enough to accept or reject the genotoxic, carcinogenic or cytotoxic potential of these fields. Up to date the International Agency for Research on Cancer (IARC) has classified the $\mathrm{X}$, gamma and ultraviolet radiation as carcinogenic and the fields generated by radio frequencies as possibly carcinogenic.
\end{abstract}

Keywords: electromagnetic fields, mutagenicity tests, cytotoxicity, magnetic phenomena, biophysical phenomena

\section{Introduction}

Electromagnetic interactions have been present in Universe since its origin. Less than a second after big bang, the electromagnetic interaction already had an effect on all existing matter. The action of such electromagnetic forces is explained as fields, which act on a volume. These fields are the result of charged particles and they affect other particles around depending on the distance between them and the magnitude of their charge.

- An electric charge causes an electric field around. The field attracts particles of its same charge or drives back opposite charges.

- An electric charge moving generates a magnetic field around.

- A magnetic field moving around a conductor material causes movement of electrons in the material (electric induction).

- A charged particle accelerated by action of a force, generates electromagnetic radiation far from the source. This radiation results of coupled magnetic and electric fields oscillating, producing a wave propagating into the space. The electromagnetic radiation involves all types of waves that do not need means of propagation, such as radio frequencies, microwaves, visible light, ultraviolet, $\mathrm{X}$ and gamma rays.

The Electromagnetic force is responsible of almost all physical, chemical and biological phenomena. In atomic scale electrons and protons interact making up elements, elements combine themselves to make molecules and molecules react producing millions of compounds and even life.

Thus, life on earth was developed under the electromagnetic fields influence. However, until the beginning of the XIX century the only electromagnetic fields on Earth were from "not human" origin, that is, Earth's magnetic field, radiation from matter and its reactions, solar and cosmic radiation. With the development of electricity and all technology depending on it, all forms of life are exposed to the electromagnetic fields generated by human made technology. 
In recent years, an interest about the effects of these fields on life, has emerged. Researches have been focused on the possible relation between electromagnetic fields and some types of cancer, their cytotoxic and genotoxic potential. In fact, the International Agency for Research on Cancer (IARC) has classified the $\mathrm{X}$, gamma and ultraviolet radiation as carcinogenic and the fields generated by radio frequencies as possibly carcinogenic. [1]

\section{Proposed mechanisms of biophysical interaction}

The form of interaction of electromagnetic fields with cells, tissues and organisms depends on a large number of variables related with the physics of the field and the biology of the organism.

Fields can be static or dynamic; they are determined by magnitude, wavelength, frequency, polarization and direction of propagation. Some mechanisms of interaction suggested by authors are briefly exposed:

\subsection{Electromagnetic induction}

Magnetic and electric fields induce electrical currents in conductors. Cells and tissues are affected by these currents, with the increase of frequencies the magnitude of the induced current increases. Strong electric fields lead to changes in orientation, rotation and movement, deformation and fusion or destruction of cells. Quantum processes are not possible, because the quantum energy of the fields is not big enough to break molecular bonds, as an ionizing radiation does ( $\mathrm{x}$, ultraviolet, gamma). [2]

Strong induced currents in tissues cause strong voltages over the cell membrane, generating a stimulation of nerve and muscle cells. The field strength needed for stimulation increases with frequency. [3]

\subsection{Penetration of electromagnetic fields}

Electromagnetic fields penetrate tissues and cells. Oscillating fields at frequencies below 10 $\mathrm{MHz}$ increase their penetration with increasing frequency. In Europe the frequency of electricity power lines is $50 \mathrm{~Hz}$. For example, the internal electric field of a person standing under a power line is approximately $10-6$ times weaker compared with the external field strength. These low frequency internal fields are not attenuated by biological tissues and their effects on health are under research. The human body presents a major absorption of energy from electromagnetic fields between 30 $\mathrm{MHz}$ to $300 \mathrm{MHz}$, range used normally in communication equipment (radio waves). The microwaves decrease their penetration with increasing frequency. [3]

\subsection{Heat generation}

The current induction in any electrical conductor generates heat. As stronger is the current induced greater is the heat absorption, and it is more important in the radio frequencies than in the low frequency ranges. [3] Protection against thermal effects of the exposure to electromagnetic fields is an issue that has concerned the authorities in order to prevent persons from excessive general or local heating. The authorities have stated limits for the specific absorption rate (SAR). SAR is a measure of the rate at which energy is absorbed by the body when exposed to a radio frequency electromagnetic field; that is the power absorbed per mass of tissue $(\mathrm{W} / \mathrm{kg})$. Heating is the predominant effect at radio frequencies above about $10-100 \mathrm{kHz}$.

\subsection{Ionic interactions}

Several types of interactions between electromagnetic fields and ions in cells and tissues have been proposed by researchers.

The first of them is the Ion - cyclotron resonance. Cyclotron resonance describes the interaction of external forces with charged particles experiencing a magnetic field, thus already moving on a circular path. Free ions move with the cyclotron frequency in magnetic fields of appropriate frequency. [4]. 
Another kind of resonance with ions is the Ion paramagnetic resonance. Electromagnetic fields of low frequency can interact with bound ions (e.g. $\mathrm{Ca}^{2+}$ ions in proteins), leading to a resonance-like behavior. The interaction with these protein-bound ions can affect the rate of binding of the ions. [2]

Electromagnetic fields can intervene in the action of ionic transport channels on membranes. Electric fields can lead to changes in the function of membrane proteins and enzymes in ion activation sites. Electromagnetic fields induce also a torque on the mineral magnetite (oxide of Iron $\mathrm{Fe}_{3} \mathrm{O}_{4}$ ) coupled to a membrane ion channel, changing the channel activity. [2]

\subsection{Spin Chemistry}

Spin chemistry is related to the interplay between spins and chemical kinetics. Basically there are two kinds of effects that follow from such interaction. First, in an external magnetic field, chemical products may be formed with distribution of magnetic spin states. Second, external magnetic fields can modify chemical yields and chemical kinetics if reaction intermediates with unpaired spins are involved. The central mechanism of spin chemistry is the radical pair mechanism with the electron spin conservation during chemical reaction steps. According to the spin conservation rule, the kind of product depends on the state of atoms (excited singlet or triplet) at the moment of the reaction. [5]

Microwave magnetic fields can lead to resonant transitions between triplet states of molecules, affecting the reaction kinetics that proceeds via triplet formation. In general, magnetic fields can influence on spin-dependent chemical reactions. [2]

In radical pair mechanism of reaction, recombination is normally only allowed in singlet states. External or internal magnetic fields can modify the electron spin states by spin evolution. As a result of the changes induced by the magnetic field in the radical pair recombination rates, the overall reaction yield is changed, affecting kinetics but not products. [2]

\subsection{Complex reaction chains}

The effects of electromagnetic fields depend on the biological-functional state of the cells, in particular on the degree of cellular activation for cells of the immune system [6]. The physical variables of the field intervene at the level of signal transduction pathways in specific cellular signaling processes.

Activation of signal pathways by external stimuli connects the physical interaction of the applied field with the biological response of the cell. The specificity of cellular processes results from different kinds of chemical and electric signals. Since electromagnetic fields don't have enough energy to penetrate the membrane directly, they may modify the existing signal transduction processes made by receptor molecules, amplifier molecules (enzymes) and even ions. [2]

\section{Possible effects of electromagnetic fields on cells}

The main interest in studying how electromagnetic fields interact with cells and their environment, is to identify the real effects of these fields in cellular function and the direct or indirect risks for life. Many authors have examined the properties and effects of electromagnetic fields in cells and tissues; they have found evidence that could demonstrate both, cytotoxicity and genotoxicity; some of them are reviewed below.

\subsection{Genotoxic effects of electromagnetic fields}

The major effect of electromagnetic fields seems to be the genotoxicity inducing the DNA damage. Some studies detected increases in micronuclei frequencies and chromosomal aberrations in samples taken from individuals exposed to low frequencies fields, such as photo-copying machine or power-line workers. Genotoxicity studies included detection of Sister Chromatid Exchange, Chromosomal Aberrations, presence of 8-hydroxy-2'-deoxyguanosine, the alkaline single cell gel electrophoresis (Comet test) and the Micronucleus test. [7] 
It is generally accepted that low frequency fields are unable to transfer energy to cells in sufficient amounts to damage DNA directly and thus, they were considered to be non-genotoxic. However, it is possible that cellular processes are altered by exposure to these fields, following the mechanisms described before. Thus, the electromagnetic fields might indirectly affect the structure of DNA, and combined with the effects in signaling and enzymatic activity, they could be correlated with cancer induction, leukemia, and fertility or genetic damages in long term. Some results showing potential genotoxicity are presented below.

Paile et al exposed human lymphocytes for 48 and 67 hours to 0.03, 0.3, $1.0 \mathrm{mT}$ magnetic fields. The data showed a significant increase in sister chromatid exchange at $1 \mathrm{mT}$, but no significant increase in chromosomic aberrations and micronuclei. [8]

Nordenson et al exposed the human amniotic cells to 0.03 or $0.3 \mathrm{mT}$ homogenous vertical magnetic field either continuously or intermittently. The observations indicated that continuous exposure for 72 hours had no effect on chromosomic aberrations, while intermittent exposure resulted in a significant increase. [9]

Ivancsits et al exposed cultures of human diploid skin fibroblasts to up to $2 \mathrm{mT}$ fields for up to 24 hours and DNA damage was assessed using the comet assay. They found a small but significant increase in DNA breakage in the cells exposed to fields as low as $35 \mu \mathrm{T}$. [10] The intermittent exposure ( $5 \mathrm{~min}$ on, $10 \mathrm{~min}$ off) was effective, while continuous exposure was not. [11] The response was dose-dependent up to $2 \mathrm{mT}$, and increased with time to a maximum after about $15 \mathrm{~h}$ of exposure. [12]

Simko et al used a tissue culture incubator with fields exposure at 0.8 and $1 \mathrm{mT}$ resulted in a significant increase in micronuclei in transformed cells but not in non-transformed cells. Researchers concluded that tumor cells are probably more sensitive to indirect effects, leading to the induction of DNA damages to chromosomal segregation failure, supporting the hypothesis that electromagnetic fields have no initiating, but possibly a promoting development of cancer. [13]

Wolf et al observed an increase in DNA breakage and formation of 8-hydroxy-2'-deoxyguanosine in leukemic cells HL-60, Rat-1 fibroblasts and WI-38 diploid fibro-blasts, after 24 and 72 hours of exposition to 0.5 and $1 \mathrm{mT}$ magnetic fields. [14]

Lai et al reported the production of single strand break and double strand break in rat brain cells after 24 hours of exposition to $10 \mu \mathrm{T}$ using the neutral comet assay procedure. [15]

Svedenstål et al exposed adult Chinese mice to $500 \mu \mathrm{T}$ electromagnetic field for 14 days. Cells collected from the front part of the brain cortex exhibited an increase in double strand break. [16] They obtained similar results in an outdoor experiment where adult mice were left in cages under electromagnetic fields generated by the $220 \mathrm{kV}$ transmission lines. [17]

Vedat et al demonstrated that exposure to high frequency fields have also genotoxic effects. They studied the bone marrow cells of mature and immature rats exposed to $1800 \mathrm{MHz}$ electromagnetic field. The field induced the genetic damage by increasing the chromosomic aberration and micronuclei. The effects were more remarkable in immature rats, normalization of DNA damage after fifteen days recovery period was observed in mature rats but not in immature rats. [18]

Yokus et al detected a significant increase in 8-hidroxy-2'-deoxyiguanosi-ne (suggestive of oxidative damage to DNA) in plasma of rats exposed to a $970 \mu \mathrm{T}$ magnetic field for 50 days. [19]

Another hypothesis, not discussed in the present review, is that the exposure to electromagnetic fields is not genotoxic itself, but it could enhance the cytogenetic damage induced by other biological, chemical, physical genotoxic agents, that is, it could have an epigenetic or non-genotoxic influence.

\subsection{Other effects of electromagnetic fields in cells.}

As it has been referred, the main effect of electromagnetic fields seems to be their genotoxicity. Some authors have studied also, other different effects of these fields, specially the influence in proliferation of cells. 
Detlavs et al demonstrated that high frequencies electromagnetic fields change the functional activity of cells. They exposed wounded regions of Wistar rats for $30 \mathrm{~min}$ daily during the first 5 days after wound infliction. They used fields of 53.53 and $42.19 \mathrm{GHz}$ without frequency modulation and $42.19 \mathrm{GHz}$ with modulation band of 200-MHz. Cells have shown alteration of their proliferative and biosynthetic properties. A field without modulation decreases inflammatory exudation and accumulation of collagenous proteins. Fields with modulation cause intensified early inflammatory exudative phenomena, and this reaction is followed by intensified synthesis of collagens. [20]

Vedat et al concluded that the in vivo exposure of mobile phone GSM-1800 $\mathrm{MHz}$ radiofrequencies at SARs $0.37 \mathrm{~W} / \mathrm{kg}$ and $0.49 \mathrm{~W} / \mathrm{kg}$ for $2 \mathrm{~h} /$ day for 45 day to rat bone marrow cells demonstrate cytotoxic and genotoxic effects in immature and mature rats. The study showed a cytotoxic effect by decreasing of the mitotic index and the ratio of polychromatic erythrocytes in bone marrow cells. [18]

Van Den Heuvel et al studied $80 \mathrm{mT}(50 \mathrm{~Hz})$ fields on haemopoietic cell proliferation. Cytotoxic effect was evaluated on $3 \mathrm{~T} 3$ cell line. The field did not cause cytotoxicity because the proliferation of the 3T3 cell line was not inhibited, but they were not able to demonstrate any interference with essential cell functions in the non-differentiated $3 \mathrm{~T} 3$ cell line. Exposure of murine bone marrow cells mice showed a reduction in the proliferation and differentiation of the granulocyte-macrophage progenitor compared to non-exposed bone marrow cells. [21]

\section{Conclusions}

Electromagnetic fields should alter biological functions, since they interact with all existing matter. A series of interaction mechanisms has been reviewed, some of them haven't been proved experimentally yet, but from a physical point of view they are well-understood, particularly as far as radical pair mechanism is concerned. In the same way, studies about direct effects of exposure of cells to electromagnetic fields have been reviewed.

The studies carried out by researchers on cells, tissues and organisms have partially demonstrated the genotoxic and possibly carcinogenic potential of such fields. However evidences found in studies in vitro and in vivo are not conclusive enough, since there are a similar number of published researches in which authors revealed that they did not find any evidence of cytotoxicity or genotoxicity.

Other studies investigated the possible carcinogenic effects of fields combined with exposure to other genotoxic agents, both chemical and physical. Positive effects have been repeatedly reported, although they have not been taken into account in this review. Electromagnetic fields could not be directly genotoxic, but they can increase the action of other genotoxic agents.

The debate about possible effects of electromagnetic fields on living organisms is still open in the scientific community, especially those due to non-thermal mechanisms of interaction with weak electromagnetic fields such as those generated by electric appliances, telecommunications and power lines.

More studies must be conducted, maintaining the same parameters of experimentation with the aim of reducing discrepancies between results, considering that the studies conducted have had differences in experimental parameters as frequency and intensity of fields, duration and mode of exposure, characteristics of the cells, tissues or organisms exposed. To date the safety of electromagnetic fields generated by all modern life appliances is doubtful and it is necessary that the public starts to take and demand more precautions in reference to exposure to electromagnetic fields on the basis of results of experimental (in vitro, in vivo) and epidemiological studies.

Acknowledgments: Universidad Antonio Narino -UAN - Colombia, Grant No. 2015049.

\section{References}

1. International Agency for Research on Cancer. IARC classifies radiofrequency electromagnetic fields as possibly carcinogenic to humans. [access 30th October 2012] Avalaible: http://www.iarc.fr/en/media-centre/pr/2011/pdfs/pr208_E.pdf 
2. Kaiser F. External signals and internal oscillation dynamics: biophysical aspects and modelling approaches for interactions of weak electromagnetic fields at the cellular level. Bioelectrochem 1996; 41: 3-18.

3. Juutilainen J, Lang S. Genotoxic, carcinogenic and teratogenic effects of electromagnetic fields. Mutat Res 1997; 387: 165-171.

4. Schwan H.P, Chiabrera in A, Nicolini C. Interactions between Electromagnetic Fields and Cells. New York: Plenum; 1985. 371p.

5. Steiner U. E, Gilch P. High magnetic fields in chemistry. In: High Magnetic Fields Science and Technology. Vol 2. Singapore: World Scientific; 2003. 288p.

6. Schimmelpfeng J, Stem J. C, Dertinger H. Action of $50 \mathrm{~Hz}$ magnetic fields on cyclic AMP and intercellular communication in monolayers and spheroids of mammalian cells. Bioelectromagnet 1995; 16: 381-386.

7. Udroiu I, Giuliani L, Ieradi L.A. Genotoxic properties of extremely low frequency electromagnetic fields. In: Non-thermal effects and mechanisms of interaction between electromagnetic fields and living matter. Vol 5. Bologna: Ramazzini Institute, European Journal of Oncology Library; 2010. 403p.

8. Paile W, Jokela K, Koivistonen A, Saloma S. Effects of sinusoidal magnetic fields and spark charges on human lymphocytes in vitro. Bioelectrochem 1995; 36: 15-22.

9. Nordenson I, Mild KH, Andersson G, Sandström M. Chromosomal aberrations in human amniotic cells after intermittent exposure to fifty hertz magnetic fields. Bioelectromagnet 1994; 15: 293-301.

10. Ivancsits S, Diem E, Pilger A, Rüdiger H.W, Jahn O. Induction of DNA strand breaks by intermittent exposure to extremely low frequency electromagnetic fields in human diploid fibroblasts. Mutat Res 2002; 519: 1-13.

11. Ivancsits S, Diem E, Pilger A, Rüdiger H.W, Jahn O. Age related effects on induction of DNA strand breaks by intermittent exposure to electromagnetic fields Mech Ageing Dev 2003; 124: 847-850.

12. Ivancsits S, Diem E, Pilger A, Rüdiger H.W, Jahn O. Intermittent extremely-low-frequency electromagnetic fields cause DNA damage in a dose-dependent way. Int Arch Occup Environ Health 2003; 76: 431-436.

13. Simkó M, Kriehuber R, Weiss D.G, Luben R.A. Effects of $50 \mathrm{~Hz}$ EMF exposure on micronucleus formation and apoptosis in transformed and nontransformed human cell lines. Bioelectromagnet 1998; 19: 85-91.

14. Wolf F.I, Torsello A, Tedesco B, Fasanella S, Boninsegna A, D'Ascenzo M et Al. 50-Hz extremely low frequency electromagnetic fields enhance cell proliferation and DNA damage: Possible involvement of a redox mechanism. Biochim Biophys Acta 2005; 1743: 120-9.

15. Lai H, Singh N.P. Magnetic field induced DNA strand breaks in brain cells of the rat. Environ Health Perspect 2004; 112: 687-694.

16. Svedenstal B.M, Johanson K.J, Hansson Mild K. DNA damage induced in brain cells of CBA mice exposed to magnetic fields. In Vivo 1999; 13: 551-2.

17. Svedenstal B.M, Johanson K.J, Mattsson M.O, Paulsson L.E. DNA damage, cell kinetics and ODC activities studied in CBA mice exposed to electromagnetic fields generated by transmission lines. In Vivo 1999; 13: $507-13$.

18. Sekeroğlu V, Akar A, Sekeroğlu ZA. Cytotoxic and genotoxic effects of high frequency electromagnetic fields (GSM $1800 \mathrm{MHz}$ ) on immature and mature rats. Ecotoxicol Environ Saf 2012; 80: 140 -144.

19. Yokus B, Cakir D.U, Akdag M.Z, Sert C, Mete N. Oxidative DNA damage in rats exposed to extremely low frequency electromagnetic fields. Free Radic Res 2005; 39: 317-23.

20. Detlavs I, Dombrovska L, Turauska A, Shkirmante B, Slutskii L. Experimental study of the effects of radiofrequency electromagnetic fields on animals with soft tissue wounds. Sci Total Environ 1996; 180: 35-42.

21. Van Den Heuvel R, Leppens H, Nêmethova G, Verschaeve L. Haemopoietic cell proliferation in murine bone marrow cells exposed to extreme low frequency (ELF) electromagnetic fields. Toxicol in Vitro 2001; 15: 351-355. 